EFFECTS OF 1988 FIRES ON AQUATIC SYSTEMS OF YELLOWSTONE NATIONAL PARK

\author{
G. Wayne Minshall \\ and \\ Christopher T. Robinson \\ Stream Ecology Center \\ Department of Biological Sciences \\ Idaho State University \\ Pocatello
}

Collections and measurements were made in september-october 1988 at 18 burn and four unburned reference sites (Table 1) and in March 1989 at 12 of the burn and one of the unburned sites under support from the National Science Foundation and the US Fish and Wildlife Service. These are being processed and analyzed as part of the present project. In addition, the original 22 burn and reference streams were examined again during August 1989 for comparison with conditions found just after the fire. With a few minor exceptions (e.g., the recording thermometers at a few sites were broken or missing) all of the information specified in our research proposal for September-October 1988 and August 1989 was obtained. Adverse weather conditions or "bear closures" prevented the assessment of all 22 sites in March but the data from the 12 sites that were sampled for invertebrates, periphyton, and organic matter appear to be sufficient to strongly suggest that changes in food quality due to the fire had an adverse effect on the benthic invertebrate community as we had hypothesized (see below).

All of the physical and chemical environment determinations (Table 2) have been completed through the tabulation and initial statistical or graphical analyses of the data, except for assessment of retention device densities from field-drawn maps. The processing of all periphyton samples (chlorophyll a and AFDM) has been completed and summary tables compiled. A total of 279 periphyton samples were collected. Transported organic matter (CPOM and FPOM) was collected at each study site (22 sites, two samples/site) in October and August 1989. All 88 samples have been processed for AFDM and characterized for type of organic material (including $\%$ charcoal).

As we had anticipated, the processing of benthic macroinvertebrate samples is only half finished at this time. 
Table 1. STREAM RESEARCH SITES FOR YELLOWSTONE NATIONAL PARK WILDFIRE STUDY $(M=$ mosaic; $B=$ complete burn; SONYEW = System of Numbering Yellowstone Waters (Mahony and Lentsch 1986; UTM = Universal Transverse Mercator Map (coordinate system)

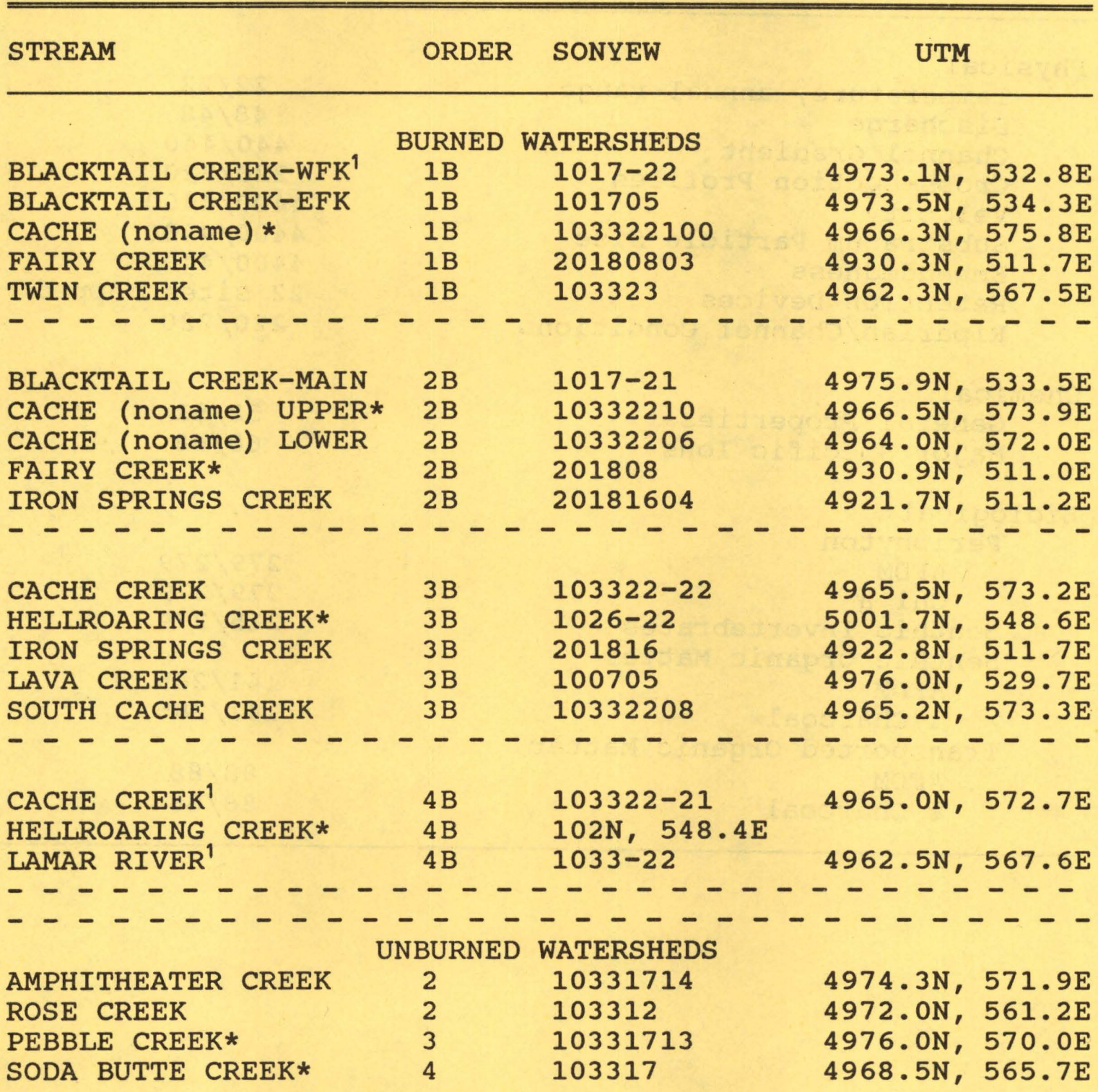

*Not sampled March 1989 due to adverse weather conditions or "bear closure".

${ }^{1}$ Sampled in March but no invertebrate collections obtained due to thick ice or high water. 
Table 2. Summary of measurments made.

Variable

Samples or Measurements Processed/Total obtained

Physical
Temperature, annual range
Discharge
Channel Gradient
Cross-section Profiles
Velocity
Substratum Particle Size
Embeddedness
Retention Devices
Riparian/Channel Conditions

Chemical

General Properties

Major Specific Ions

Biological

Periphyton

AFDM

$\mathrm{Chl}$ a

Benthic Invertebrates

Benthic Organic Matter

AFDM

\% Charcoal

Transported Organic Matter

AFDM

\% Charcoal
$22 / 22$

$48 / 48$

$440 / 440$

$220 / 220$

$4400 / 4400$

$4400 / 4400$

$4400 / 4400$

22 sites mapped

$220 / 220$

$52 / 52$

$68 / 68$

$279 / 279$

$279 / 279$

$215 / 279$

$141 / 285$

$141 / 285$

$88 / 88$

$88 / 88$ 
The study protocol involved the collection of 110 samples from 22 sites (five/site) each collected in october 1988 and August 1989, and 59 samples from the 12 sites (1 missing from WF Blacktail Deer Cr.) collected in March 1989. Of these 279 samples, 215 have been hand sorted (to separate macroinvertebrates from plant matter and inorganic sediments) and 145 have been processed completely (invertebrates identified, enumerated, and weighed). Macroinvertebrate data for two samples from 10 sites each from October 1988 and March 1989 are included in this report. Once the macroinvertebrates have been removed from the benthic sample, the remaining material associated with that sample is characterized in terms of the relative composition of plant organic matter. This material is then dried, weighed, ashed, and reweighed, to determine organic mass.

\section{SIGNIFICANT FINDINGS}

Biological Relationships. Between October 1988 and March 1989 there were marked decreases in abundance, richness, and H' diversity in six of eight burned sites examined, whereas these values increased or remained constant in the unburned streams. We attribute these changes to high amounts of charcoal on the stream bottoms and in transport as a result of the fires, since no physical disturbances from the runoff occurred during this period. We believe that the charcoal decreased the palatability and quality (e.g., increased $C: N$ values) of the organic matter pool as food. Although benthic organic matter increased in amounts between October and March in six burned streams, it decreased in five others and similar patterns were seen in the two unburned streams, however, the burned streams all had substantially greater proportions of charcoal making up the BOM than did the reference streams and in all but three of the burned streams the amounts of charcoal increased while decreasing in the unburned streams (except Iron Springs $3^{\circ}$ ) during this period, with comparable changes observed in the unburned streams.

Transported organic matter (TOM) was collected in October 1988 and August 1989 from all study streams and categorized as coarse particulate (CPOM) or fine particulate organic matter (FPOM). These data indicate that $67 \%$ of the burn sites showed an increase in the amount of CPOM between October and August whereas only $25 \%$ of the unburned showed an increase for the same period. Charcoal contributed greater than $50 \%$ of the CPOM mass in the burn sites while averaging less than $10 \%$ of the mass of the unburned sites. The relative amount of charcoal in the CPOM was similar between years at most burn sites. 
The quantity of FPOM was substantially greater in August 1989 than in october 1988 (from two to about 100x) in $90 \%$ of the burn sites, although the Upper Cache Creek site showed a several-orders-of-magnitude increase. Only $25 \%$ of the unburned sites showed an increase in FPOM in August 1989 over october 1988. As with CPOM, the relative amount of charcoal in the FPOM was higher in the burn sites (usually greater than $50 \%$ ) than in the unburned sites (generally $0 \%$ ). In addition, the percent charcoal typically was higher in August 1989 than in October 1988 in the burn sites.

Physical and Chemical Relationships. Melting of the spring 1989 snow pack was much slower than had been anticipated (P. Farnes, SCS, Bozeman, pers. comm.). Consequently, even though several periods of "blackwater" associated with runoff from heavy rains occurred between runoff and our August sampling period, erosion of the stream bed and alteration to channel morphology generally were much less than we had anticipated. However, several 1st and 3rd order streams, particularly, in the Cache Creek and Hellroaring Creek drainages, did sustain substantial channel alteration. Should a normal or above snowpack develop this winter and more usual runoff patterns occur this spring, severe scouring of the channels may still develop. But, if the milder conditions experienced in 1989 persist over the next year of so, damage from the fire may be much less severe and recovery much more rapid than we had originally predicted (Minshall et al. 1989). Even with the more "controlled" runoff, changes in channel conditions were detected. Marked reductions were observed in diversity of current velocities in the burned streams, as indicated by differences in coefficients of variation between the two dates. Similar changes in substratum were recorded in half of the burned stream sites. No comparable changes in either variable occurred in the unburned streams. Regression analysis between substratum axes measured across sites indicate that a single rock dimension is adequate to describe the substratum particle-size characteristics for a site. In addition, estimates of required sample size indicate that an $n=100$ rocks is adequate for characterizing substratum particle size at a site at $90 \%$ confidence level.

\section{PROBLEMS THAT MAY IMPEDE PERFORMANCE AND CORRECTIVE ACTION}

The greatest problem associated with most stream studies involving field research is the intensive laboratory time needed to work-up samples. Benthic samples alone require an average of $5 \mathrm{~h}$ to process. Once processed, the raw data needs to be computerized for reduction and analysis. The data- 
reduction step also is a time consuming process due to large number of taxa (averaging 20-40 per site) found in lotic benthic samples. However, once the data are entered and reduced to means and standard deviations, the analysis roceeds relatively quickly. In order to speed up the initial processing steps, we have added personnel to this stage but even this requires time for proper training and supervision. 